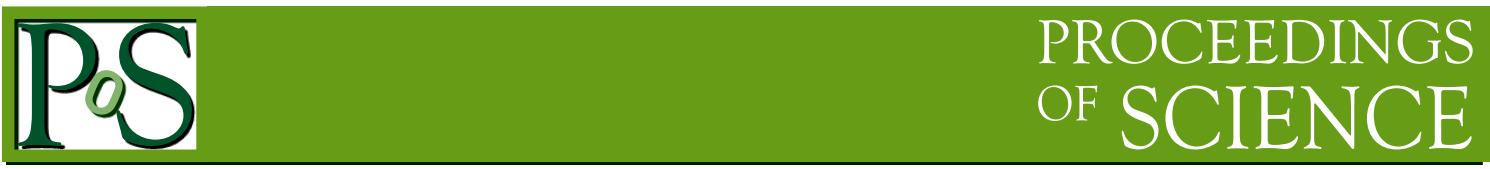

\title{
No Low-Lying Nuclear Vibrations: Configuration Dependent Pairing and Axial Asymmetry
}

John F Sharpey-Schafer ${ }^{1}$

University of Western Cape

Department of Physics, P/B X17, Belleville, ZA-7535, South Africa

E-mail: jessetlabs.ac.za

The present wealth of experimental data on the structure of deformed atomic nuclei challenges the original interpretation by Bohr and Mottelson of low-lying excited $\mathrm{K}^{\pi}=0^{+}$and $\mathrm{K}^{\pi}=2^{+}$rotational bands as $\beta$ and $\gamma$ time-dependent vibrations of the quadrupole shape. It is shown that the first excited $\mathrm{K}^{\pi}=0_{2}{ }^{+}$bands are pairing isomers with seniority zero lowered into the pairing gap by configuration dependent pairing to form a second vacuum (SV) analogous to the ground state vacuum. These bands are $2 \mathrm{p}-2 \mathrm{~h}$ single particle configurations and have nothing to do with nuclear "vibrational collectivity". In contrast, the first excited $\mathrm{K}^{\pi}=2^{+}$bands, that are found in all quadrupole deformed nuclei, are collective and exist as a consequence of the breaking of axial symmetry.

54th International Winter Meeting on Nuclear Physics

25-29 January 2016

Bormio, Italy

\section{${ }^{1}$ Speaker}




\section{Introduction}

For many years the most authoritative source of information with which to understand the details of nuclear spectroscopy has been Bohr and Mottelson's famous two volumes of "Nuclear Structure" [1]. On page 363 of Vol II they explain the concept of time-dependent vibrations of the quadrupole deformed nuclear shape: orthogonal oscillations may take place (a) along the symmetry axis giving a variation in time of the axial deformation $\beta$, the so-called $\beta$ vibrations (b) perpendicular to the symmetry axis, breaking the axial symmetry, the $\gamma$ vibrations. The former are identified in actual even-even deformed nuclei with the first excited $\mathrm{O}_{2}{ }^{+}$state which is the band head of the first non-yrast $\mathrm{K}^{\pi}=0^{+}$rotational structure. The latter is identified with the first $\mathrm{K}^{\pi}=$ $2^{+}$band which is also found in all deformed even-even nuclei.

In even-even deformed nuclei the strong attractive force between nucleons ensures that like nucleons in time reversed orbits (with magnetic quantum numbers $m$ and $-m$ ), having wave functions with maximum overlap, come closest to each other and hence have lower energies than other options. This "pairing" interaction ensures that the ground states of all even-even nuclei, spherical or deformed, have spin $0_{1}{ }^{+}$without exception. This "pairing energy" is typically $\sim 1.0 \mathrm{MeV}$ per nucleon in the middle of the nuclear chart for nuclei with atomic mass $A=150$ [1] (vol. I p170). To break a pair requires approximately $2.0 \mathrm{MeV}$ meaning that particle-hole (p-h) states will only occur at excitation energies of $\sim 2.0 \mathrm{MeV}$ and greater.

Up to an excitation energy of $2.0 \mathrm{MeV}$ the density of states in the residual even-even nucleus is low. After $2.0 \mathrm{MeV}$ there is suddenly a very high density of excited states due to p-h channels opening up. However there are some states in the "pairing gap" between the ground $0_{1}{ }^{+}$paired state at $0.0 \mathrm{MeV}$ and the onset of $\mathrm{p}-\mathrm{h}$ states at $\sim 2.0 \mathrm{MeV}$. Some of these states are just the rotational excitations of the intrinsic states located in the pairing gap. The traditional interpretation of the intruder intrinsic states, that we have taught all our students, is that they must be due to "collective" excitations of the nucleus. Obvious possible collective in-phase motions of the nucleons are time-dependent vibrations of the nuclear shape, in line with the "liquid drop" model of the nucleus, proposed by George Gamow [2] and advocated by Nils Bohr [3,4].

\section{Classical Considerations}

For a classical liquid drop the dynamic properties of such a drop, assuming irrotational flow, had been formulated by Lord Rayleigh (John William Strutt) in 1879 [5]. Assuming an inviscid incompressible liquid sphere he obtained:

$$
\omega^{2}=(\lambda-1) \lambda(\lambda+2) \gamma / \rho R^{3}
$$

where $\omega$ is the frequency of the $\lambda$ pole oscillation of the drop, $\gamma$ is the surface energy per unit area due to the surface tension, $\rho$ is the density of the liquid and $R$ is the radius of the spherical drop. That $\omega^{2} \propto \gamma / \rho R^{3}$ is easily obtained by elementary dimensional analysis. The application of this to a charged spherical nucleus, also assuming no viscosity and irrotational flow, gives [6]:

$$
\omega^{2}=\left\{(\lambda-1) \lambda(\lambda+2) C s / 3 R_{A}{ }^{2} m A\right\}-\left\{2(\lambda-1) \lambda e^{2} Z^{2} /(2 \lambda+1) 4 \pi \epsilon_{0} R_{A}{ }^{3} m_{p} A^{2}\right\}
$$

where the radius of the nucleus $\mathrm{R}_{0}=\mathrm{R}_{\mathrm{A}} \mathrm{A}^{1 / 3}$, Cs is the surface energy term in the Weizsäcker Mass Formula $\sim 18 \mathrm{MeV}$ [7], $\mathrm{m}$ is the nucleon mass, $\mathrm{A}$ is the atomic mass number, the second term is due to a uniform charge $\mathrm{Ze}^{+}$spread throughout the nucleus and $\mathrm{m}_{\mathrm{p}}$ is the mass of the proton. The second term has little effect on $\omega$ if $Z<80$. Quantising by putting $E_{\lambda}=\hbar \omega$ for $A=150$ and $\lambda=2$ the Rayleigh term gives $\mathrm{E}_{2} \approx 2.4 \mathrm{MeV}$ and for $\lambda=3, \mathrm{E}_{3} \approx 4.6 \mathrm{MeV}$.

However, we know from the Strutinski [8,9] shell corrections to the liquid drop that the shell corrections oscillate much more rapidly than the liquid drop energy with increasing quadrupole 
deformation. Hence, for any deformed nucleus, the potential it sees, with respect to quadrupole deformation, will be more constrained than the liquid drop which will increase any vibrational frequency and the Rayleigh vibrational excitation energy. In 1941 Flügge [10] used the Rayleigh formula to estimate the energy of the lowest vibrational mode in nuclei. The formula was quantised in 1952 by Aage Bohr [11], to give his famous Bohr Hamiltonian, using the Dirac prescription [12,13] for replacing time differentials of canonical variables with their space differentials multiplied by -ih. The main problem with this approach is that it is not at all clear what the value of the mass parameter $B$ should be that is crucial in working out any vibrational excitation energies. Like Raleigh, Aage Bohr assumes the nuclear "liquid" is irrotational. But we know that the moments-of-inertia $\mathscr{I}$ deduced from the measured quadrupole moments and the energy spacing of rotational bands, is between the irrotational and the rigid body values. The increase of $\mathscr{I}$ from irrotational towards rigid, will surely increase any vibrational frequency of the shape? The quantity $B$ is usually fitted to the data assuming that the first excited $\mathrm{K}^{\pi}=0^{+}$and $\mathrm{K}^{\pi}=2^{+}$bands are indeed $\beta$ and $\gamma$ vibrations.

\section{3. $\mathrm{K}^{\pi}=\mathrm{O}^{+}, \mathrm{O}_{2}{ }^{+}$states}

The experimental properties of excited $\mathrm{K}^{\pi}=\mathrm{O}^{+}, \mathrm{O}_{2}{ }^{+}$bands in even-even deformed nuclei have been reviewed by Paul Garrett [14]. He points out that most of these bands do not have the attributes required of $\beta$ vibrations, including the $\mathrm{B}(\mathrm{E} 2)$ transition rates to the ground state band. He also stresses "...the need to consider the role of pairing in the description of these states." The nuclei that came nearest to satisfying the Bohr and Mottelson criteria for a collective $\beta$ vibration were nuclei near the ${ }^{154} \mathrm{Gd} \mathrm{N}=90$ nucleus. Bohr and Mottelson are clear about the meaning of a vibration; "A vibrational mode of excitation is characterized by the property that it can be repeated a large number of times. The nth excited state of a specified mode can thus be viewed as consisting of n individual quanta. The quanta obey Bose statistics..." [1].

A very beautiful experiment by Kulp et al. [15] clearly demonstrates that there are no candidate two phonon $0_{\beta \beta}{ }^{+}$states in the $\mathrm{N}=90$ nucleus ${ }^{152} \mathrm{Sm}$. The $0_{2}{ }^{+}$states themselves, in the $\mathrm{N}=88$ and 90 nuclei, lie at low excitation energies of less than $900 \mathrm{keV}$. They are populated strongly in two neutron transfer reactions when the transfer is across $\mathrm{N}=89$. In the ${ }^{152} \mathrm{Gd}(\mathrm{t}, \mathrm{p}){ }^{154} \mathrm{Gd}$ reaction [16] the $0_{2}{ }^{+}$state is very strongly populated and the $0_{3}{ }^{+}$state and $0_{4}{ }^{+}$states are also fairly strongly populated. This demonstrates that these $0_{\mathrm{n}}{ }^{+}$states have significant amounts of paired two neutrons outside the target core in their wavefunctions. Unfortunately, two nucleon stripping only gives information on the spin and parity of the final state and not on the specific single particle orbital involved. Single particle transfer does not populate these $\mathrm{O}_{2}{ }^{+}$states with sufficient strength to give any information on the angular momentum of the transferred nucleon.

However, the intrinsic configuration of any core excitation of an even-even nucleus can be coupled to by the odd nucleon, or nucleon hole, in the neighbouring odd nuclei as long as that nucleon is not Pauli blocked by the core excitation having a time-reversed pair of nucleons in the same orbital. We therefore have to look for the orbital that does not couple to the core excitation. Schmidt et al. [17] have made a very comprehensive study of the low-spin states of ${ }^{155} \mathrm{Gd}$ using the $(n, \gamma)$ reaction and $(d, p)$ and $(d, t)$ neutron transfer reactions. Candidates for the coupling of the low-K orbitals to the ${ }^{154} \mathrm{Gd} \mathrm{O}_{2}{ }^{+}$state are found. Their experiments populate states in ${ }^{155} \mathrm{Gd}$ of low spin and only weakly populate the [505]11/2- isomer at $121 \mathrm{keV}$ with $\mathrm{L}=5$ in the transfer reactions and none of the higher spin states above it. Their assignment of the $592 \mathrm{keV}$ level as the [521]3/2- 
neutron coupled to the $0_{2}{ }^{+}$state in ${ }^{154} \mathrm{Gd}$ is elegantly supported by the data from the ${ }^{157} \mathrm{Gd}(\mathrm{p}, \mathrm{t}){ }^{155} \mathrm{Gd}$ reaction [18].

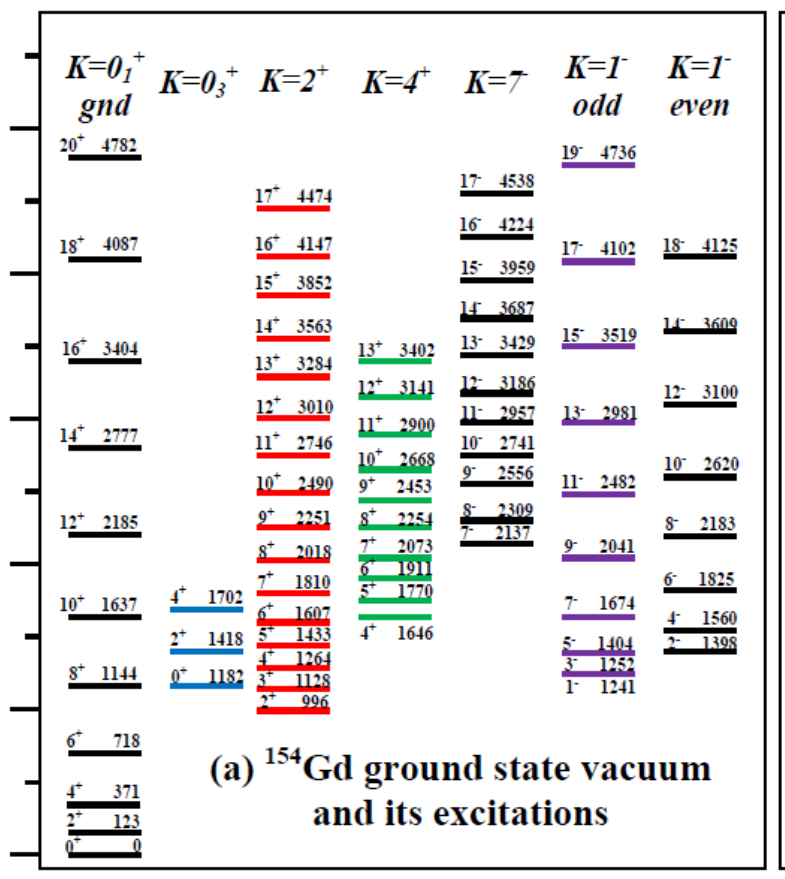

$\begin{array}{r}\mathrm{KV}_{2} \mathrm{O}_{2}^{+} \\ \mathrm{SV}\end{array} \quad \mathrm{K}=0_{7}^{+} \quad \mathrm{K}=2^{+} \quad \mathrm{K}=4^{+}$others

(b) ${ }^{154}$ Gd second vacuum and its excitations lowered by

$\begin{array}{lll}20^{*} & 4647 & 681 \mathrm{keV}\end{array}$
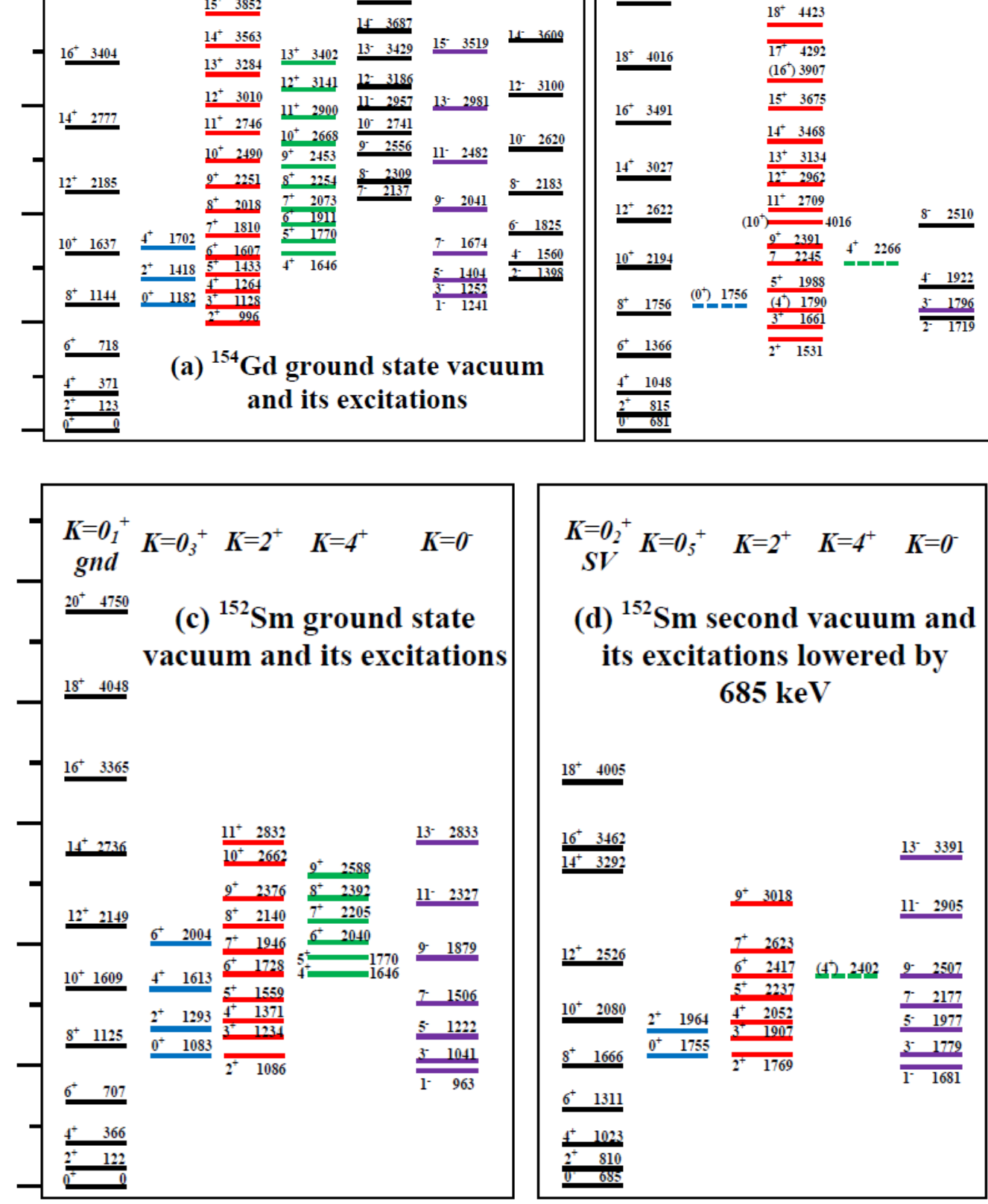

$\underset{\mathrm{SV}}{=\mathrm{O}_{2}^{+}} \mathrm{K}=0_{5}^{+} \quad \mathrm{K}=2^{+} \quad \mathrm{K}=4^{+} \quad K=0^{-}$

(d) ${ }^{152} \mathrm{Sm}$ second vacuum and its excitations lowered by $685 \mathrm{keV}$

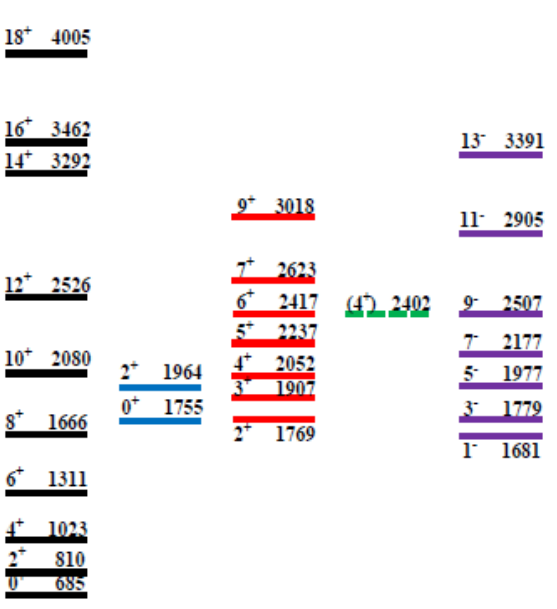

Fig. 1 Rotational bands in ${ }^{154} \mathrm{Gd}[21]$ and ${ }^{152} \mathrm{Sm}$ [20], allocated to their respective vacua, to demonstrate their congruence. Bands allocated to (a) the ground state vacuum $\left|0_{1}{ }^{+}\right\rangle$in ${ }^{154} \mathrm{Gd}$ (b) the second vacuum $\mid \mathrm{O}_{2}{ }^{+}>$in ${ }^{154} \mathrm{Gd}$. These latter states have been lowered by $681 \mathrm{keV}$ so that $0_{1}{ }^{+}$and $0_{2}{ }^{+}$are at the same height in the figure [19]. (c) and (d) the same for ${ }^{152} \mathrm{Sm}$. 
Our ${ }^{154} \mathrm{Sm}(\alpha, 3 \mathrm{n}){ }^{155} \mathrm{Gd}$ experiment at iThemba LABS using the AFRODITE $\gamma$-ray spectrometer showed that the [505]11/2- neutron does not couple to the $0_{2}{ }^{+}$state in ${ }^{154} \mathrm{Gd}$ [19]. This indicates that the core $0_{2}{ }^{+}$state in ${ }^{154} \mathrm{Gd}$ has two [505]11/2- neutrons as a major component of its configuration. The [505]11/2- neutron is Pauli blocked from coupling to the core $\mathrm{O}_{2}{ }^{+}$state. Clearly the same blocking should occur for all the [505]11/2- neutrons outside even-even cores in the $\mathrm{N}=88$ and 90 nuclei. Indeed this blocking situation exists in all other $\mathrm{N}=89$ and 91 neighbouring odd neutron nuclei.

If the $\mathrm{O}_{2}{ }^{+}$states are 2 neutron-2hole seniority zero states, they form a second vacuum (SV) analogous to the ground state in which all nucleons are paired. Hence they should have all the structures built upon them that the ground state has. In the $\mathrm{N}=90$ nuclei ${ }^{152} \mathrm{Sm}[20]$ and ${ }^{154} \mathrm{Gd}[21]$ the level schemes may be divided into two congruent sets of levels, one set based on excitations of the ground state, the other on excitations of the second vacuum $\left(\mathrm{SV}, \mathrm{O}_{2}{ }^{+}\right.$state). The congruence of the excitations built on the $\mathrm{O}_{2}{ }^{+}$states with respect to their ground state excitations is remarkable, Fig. 1. The structures belonging to both vacua in both nuclei include $\mathrm{K}^{\pi}=2+$ bands and negative parity bands.

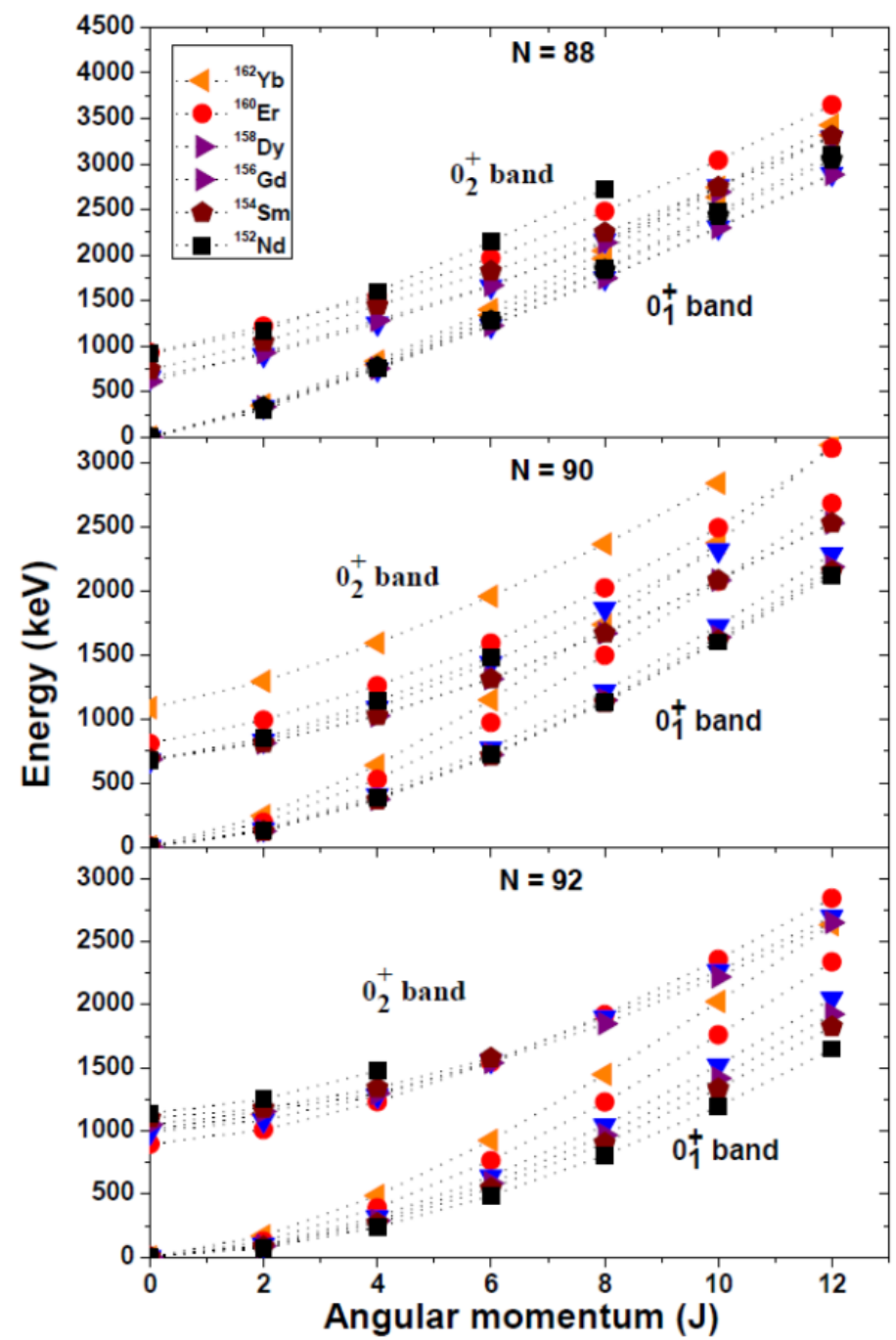

Fig. 2. Excitation energy as a function of angular momentum for members of the ground state $0_{1}{ }^{+}$bands and the $\mathrm{O}_{2}{ }^{+}$bands for the $\mathrm{N}=88,90$ and 92 isotones [22]. The deformation of $0_{1}{ }^{+}$ground state bands decrease as the proton number $Z$ increases, whereas the deformation of the excited $0_{2}{ }^{+}$bands does not. In all IBM calculations the moment-of-inertia for the $0_{2}{ }^{+}$bands are less than for the ground state $0_{1}{ }^{+}$bands [24]. 
Recent measurements at iThemba LABS have identified the $\mathrm{K}^{\pi}=0_{2}{ }^{+}$bands in ${ }^{158}{ }_{68} \mathrm{Er}_{90}$ [22] and ${ }^{160}{ }_{70} \mathrm{Yb}_{90}$ [23] to higher spins than seen in $\beta$-decay experiments. These measurements allow the comparison of the behaviour of these bands with spin with that of the ground state yrast $\mathrm{K}^{\pi}=$ $0_{1}{ }^{+}$bands as the proton number increases and the deformation decreases. This comparison is shown in Fig. 2 where the excitation energies are plotted against spin for $\mathrm{Z}=60$ to $70{ }_{60} \mathrm{Nd}$ to ${ }_{70} \mathrm{Yb}$ ) for neutron numbers $\mathrm{N}=88,90$ and 92. It is clear that the ground state $\mathrm{K}^{\pi}=0_{1}{ }^{+}$bands are decreasing in deformation, as the proton number $\mathrm{Z}$ increases after ${ }^{156}{ }_{66} \mathrm{Dy}_{90}$, while the $\mathrm{K}^{\pi}=\mathrm{O}_{2}{ }^{+}$ bands maintain an almost constant moment-of-inertia. This is in contrast to the predictions of all IBA and similar models where the moments-of-inertia of the excited $\mathrm{K}^{\pi}=\mathrm{O}_{2}{ }^{+}$bands are always less than the moments-of-inertia of the ground state $\mathrm{K}^{\pi}=0_{1}{ }^{+}$bands. To quote [24] "While the IBA calculations using the most common form of the IBA Hamiltonian reproduce the energetics of the $\mathrm{O}_{2}{ }^{+}$mode, they fail to account for the properties of the states built upon it."

\section{Pairing Isomers}

Clearly the $0_{2}{ }^{+}$states in $\mathrm{N}=88$ and 90 nuclei are not due to $\beta$ vibrations of the nuclear shape. But if they are 2 neutron 2 neutron-hole states, how is it that they can be lowered so far into the pairing gap? The solution was found in the early 1970s and applied to the $0_{2}{ }^{+}$states in $\mathrm{Th}, \mathrm{U}$ and $\mathrm{Pu}$ nuclei. These had been observed in (p,t) two neutron pick-up reactions by Maher et al. [25] but not in (t,p) two neutron stripping reactions by Casten et al. [26]. The solution put forward by Griffin, Jackson and Volkov [27] was that simple monopole pairing was too crude an approximation to explain excited $0_{n}{ }^{+}$states. With monopole pairing all the two particle transfer strength is decanted into the ground state [28]. Clearly this is not the case for actinide nuclei or for the $\mathrm{N}=88$ and 90 nuclei discussed above. Ref. [27] postulate that in the pairing model scattering from one pair of time-reversed orbits to another pair of time-reversed orbits the probability increases with the minimization of the momentum transfer in the process and with the overlap of the wavefunctions of the initial paired orbit with the wavefunctions of the final paired orbit. Thus nucleons in low- $\Omega$ Nilsson orbits are inhibited from scattering into high- $\Omega$ orbits. Nucleons in low- $\Omega$ Nilsson orbits have positive (prolate) single particle quadrupole moments whereas nucleons in high- $\Omega$ orbits have negative (oblate) quadrupole moments. Hence a better approximation to the pairing interaction is one that depends on the quadrupole moment of the nucleons involved: hence "quadrupole pairing".

To demonstrate the effect of this improvement in the pairing interaction Ref. [27] has a convincing toy model: Suppose that $\Delta_{\mathrm{pp}} \approx \Delta_{\mathrm{oo}} \gg \Delta_{\mathrm{op}}$ where $\Delta_{\mathrm{pp}}, \Delta_{\mathrm{oo}}$, and $\Delta_{\mathrm{op}}$ are the pairing interactions between nucleons scattering between prolate-prolate, oblate-oblate and prolate-oblate Nilsson orbitals. Also suppose there are $n$ prolate and $n$ oblate orbitals at the Fermi surface. Assume that each pairing matrix element is the same for the same type $-a$, BUT the prolate-oblate matrix elements are very weak $-\varepsilon a$. Then if the prolate $n^{*} n$ matrix is $A$, the oblate matrix is also $A$, the matrix for the total system is;

$$
\left\{\begin{array}{lr}
A & \varepsilon A \\
\varepsilon A & A
\end{array}\right\}
$$

Then there are (2n-2) states with ZERO energy and 2 states with spin $0+$ and energies $E_{1,2}=-(1 \pm$ $\varepsilon) n a$ and separated by an energy of $2 \varepsilon n a$. Obviously there is mixing between the two lowered $0^{+}$ states depending on the size of $\varepsilon$. 
It was pointed out by Abdulvagabova, Ivanova and Pyatov [29] that in reality the matrix (2) is not symmetric in the density of prolate and oblate states. High- $\Omega$ oblate states are extruded to the Fermi surface at the onset of deformation but have a much lower density of states than the prolate low- $\Omega$ states that are driving the deformation. Hence the pairing interaction for oblate orbitals will be much reduced compared to the pairing involving prolate orbitals. Hence the prolate paired state will be the $0_{1}{ }^{+}$ground state and the oblate paired state will be the excited $\mathrm{O}_{2}{ }^{+}$state. These authors [29] also pointed out that these conditions also applied in the $\mathrm{N} \sim 90$ nuclei as well as in the actinides. Other authors have also developed this quadrupole pairing model [30-32]. The latter authors coined the term "pairing isomers" for these $0_{2}{ }^{+}$states.
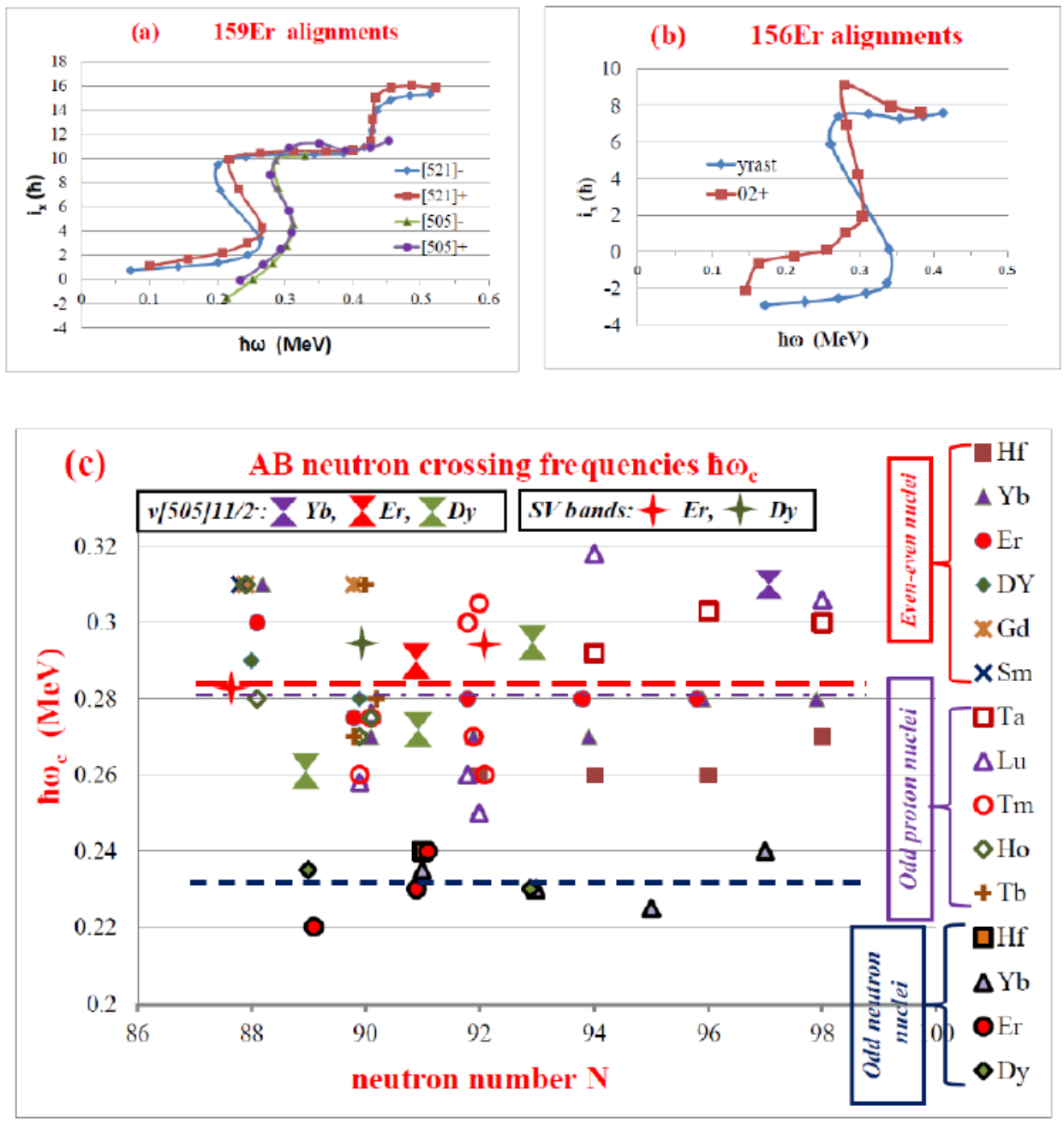

Fig. 3. Alignments of bands in (a) the odd-nucleus ${ }^{159} \mathrm{Er}_{91}$ showing the reduction of the critical alignment frequency $\hbar \omega_{c}$ due to blocking of the monopole pairing in both signatures of the ground state [521]3/2- band compared to the lack of blocking in both signatures of the [505]11/2- band. (b) the even-even nucleus ${ }^{156} \operatorname{Er}_{88}$ showing that the $\mathrm{O}_{2}{ }^{+}$band has the same unblocked critical alignment frequency as the yrast band. (c) critical frequencies $\hbar \omega_{\mathrm{c}}$ for the alignments of the $\mathrm{AB}$ "back-bends" due to $i_{13.2}$ neutrons in a variety of rotational bands in nuclei with neutron numbers between $\mathrm{N}=88$ and 98 . The long dashed red line is the average $\hbar \omega_{c}$ for even-even nuclei, the mauve dashed-dotted line is the average $\hbar \omega_{c}$ for odd proton nuclei and the dark blue short dashed line is the average $\hbar \omega_{c}$ for odd neutron nuclei. Clearly the odd neutron [505]11/2- nuclei and the Second Vacuum (SV) $0_{2}{ }^{+}$band alignments do not suffer the same reduction in $\hbar \omega_{\mathrm{c}}$ that the other odd neutron bands do. Errors on the data points are between 5 and $10 \mathrm{keV}$. Data with larger errors have not been included. 
It is clear that the [505]11/2 neutrons will not partake in the monopole pairing if the conjecture of Ref. [27] is correct. Indeed this is found to be the case. In 1982 Jerry Garrett et al. [33] pointed out that the [505]11/2- neutron bands in the odd neutron nuclei near N 90 "back-bend" at a critical frequency of $\hbar \omega_{\mathrm{c}} \approx 0.28 \mathrm{MeV}$, which is the same unblocked frequency as the "backbends" in the neighbouring even-even and odd proton nuclei yrast bands. In contrast, in odd neutron nuclei, the neutrons in low- $\Omega$ Nilsson orbits block some of the monopole (prolate) pairing giving a "back-bending" critical frequency for the energy required to align a pair of $i_{13 / 2}$ neutrons of $\hbar \omega_{\mathrm{c}} \approx 0.23 \mathrm{MeV}$. Fig. 3(a) shows the alignments of the positive and negative signatures of the [505]11/2 band compared with those of the [521]3/2 ground state band in ${ }^{159} \mathrm{Er}$. Fig. 3(b) shows the alignments of the yrast band and the $0_{2}{ }^{+}$band in ${ }^{156} \mathrm{Er} \mathrm{[34].} \mathrm{The} \mathrm{systematics} \mathrm{of} \mathrm{current} \mathrm{data} \mathrm{on}$ $i_{13 / 2}$ neutron "AB" alignment frequencies for nuclei with $\mathrm{N}=88$ to 98 are shown in Fig. 3(c). The horizontal broken lines in Fig. 3(c) indicate the average critical frequencies for even-even, odd proton and odd neutron nuclei. The few examples of the critical frequencies in [505]11/2 odd neutron bands are marked with hour-glass symbols. It is clear that the AB critical frequency is not blocked by the [505]11/2 neutron orbital. The two known examples of $\mathrm{AB}$ alignments in $\mathrm{O}_{2}{ }^{+}$ bands are marked in Fig. 3(c) with a star symbol. Again there is no reduction of the critical alignment frequency which means that, whatever the configuration of the $\mathrm{O}_{2}{ }^{+}$state is, the neutrons involved in the configuration do not partake in the monopole pairing.

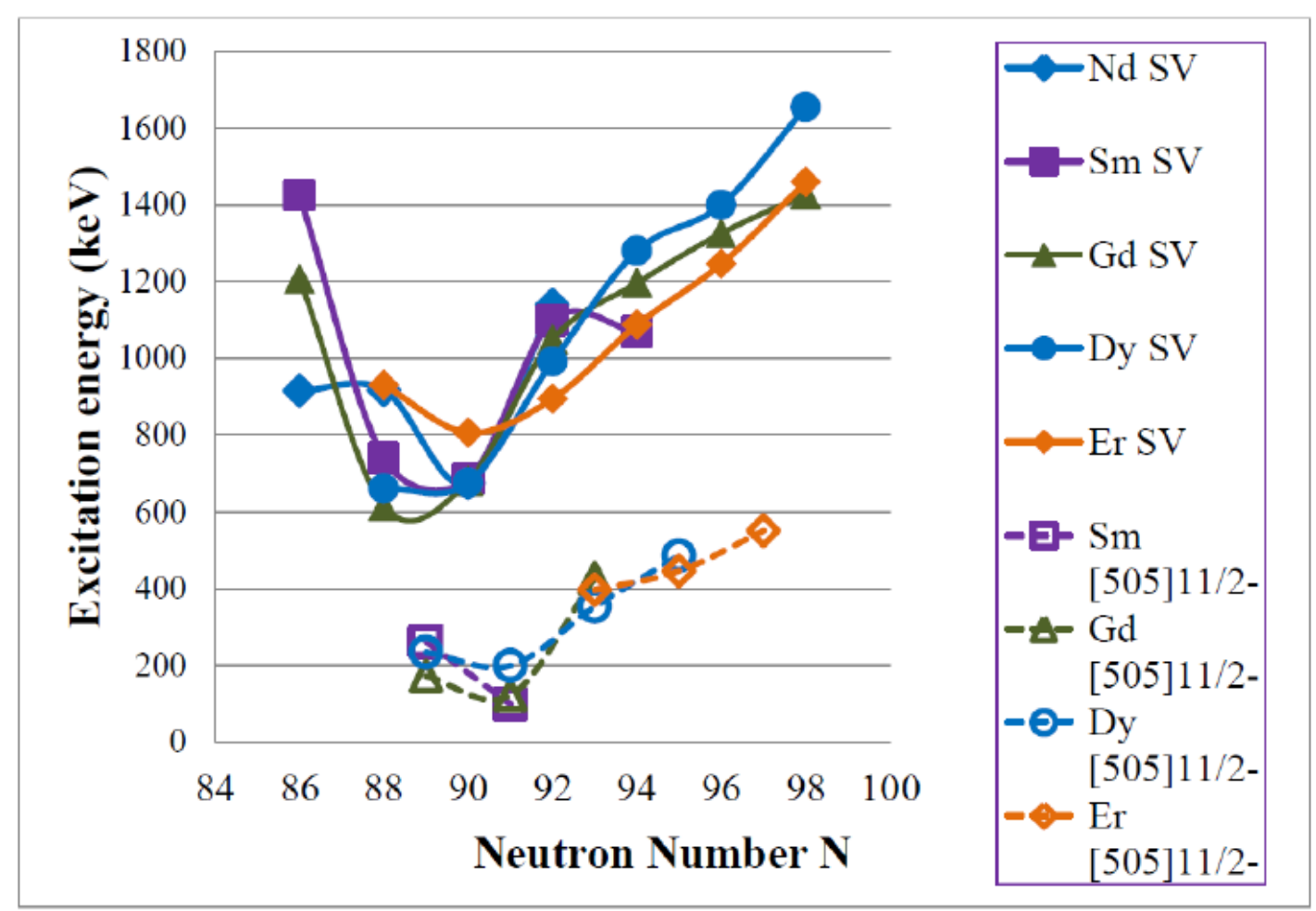

Fig. 4. Systematics of bandhead energies for [505]11/2 and $K^{\pi}=O_{2}{ }^{+}(\mathrm{SV})$ states in $\mathrm{N}=86-98$ and $\mathrm{Z}=60$ 70 nuclei.

In Fig. 4 we compare the excitation energies of the $0_{2}{ }^{+}$states and [505]11/2- single neutron states for nuclei with neutron numbers $N=88-98$ and proton numbers $Z=60-68$. The strong correlation between the two sets of energies is manifest. We draw an analogy between the trajectory of the [505]11/2 neutron orbital, with respect to the Fermi surface, and that of a "flying fish"! 
The [505]11/2 orbital is chased to the surface by the increasing nuclear deformation as the neutron number is increased, Fig 5. It then flops back into the Fermi Sea as orbitals get filled by further increases in the neutron number. As the [505]11/2 orbital retreats from the Fermi surface the excitation energy of the $\mathrm{O}_{2}{ }^{+}$states increase, driving them nearer the point where the $\mathrm{p}$-h states start. This will lead to an increase in mixing with other configurations.

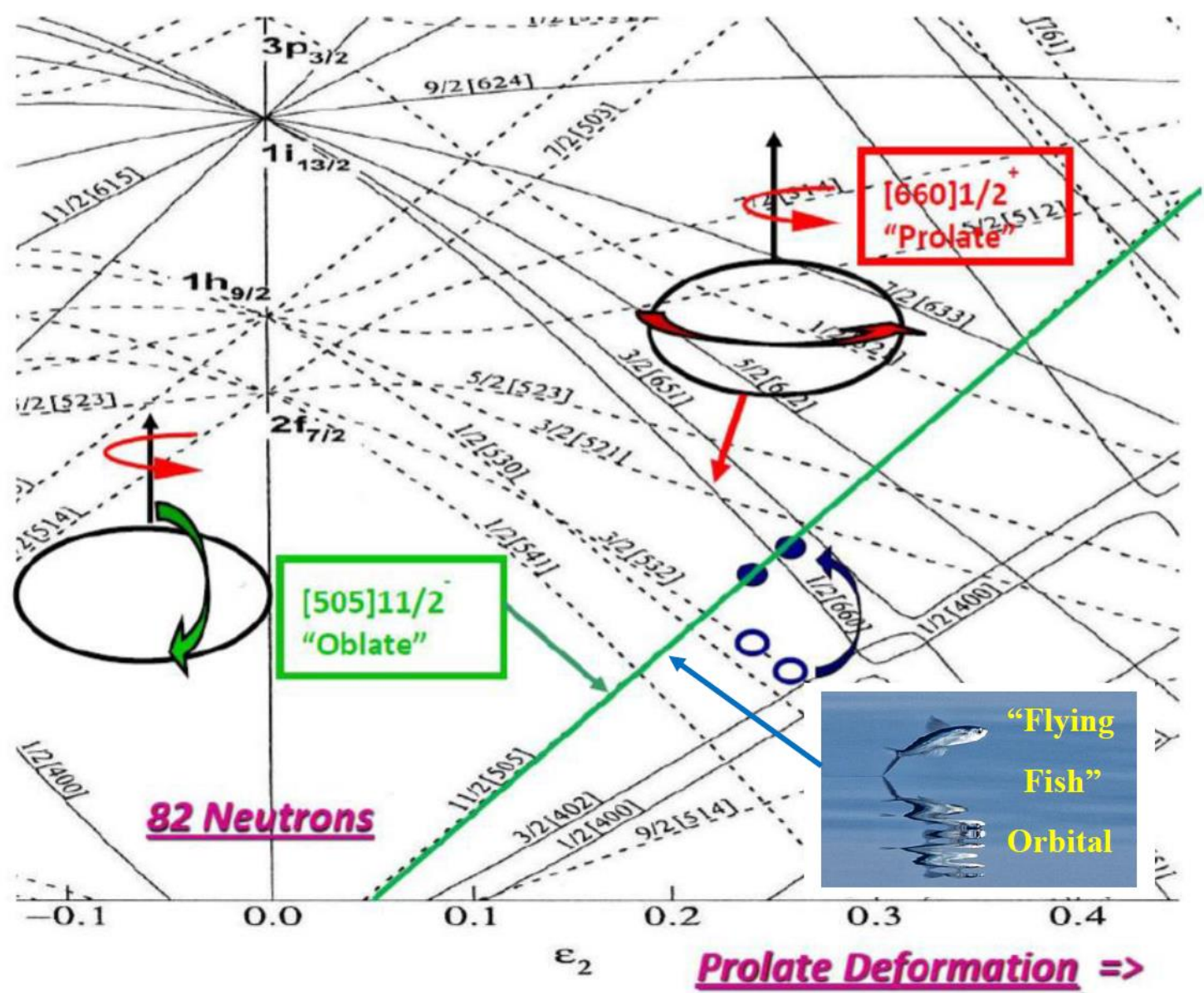

Fig. 5. Detail of the Nilsson diagram showing orbitals determining the structure of nuclei with neutron number $N>82$. Prolate deformation brings down low- $\Omega$ orbits from the single particle levels above the $N$ $=82$ shell gap and extrudes high $-\Omega$ levels to the Fermi surface from the high- $j$ single particle levels below the gap. Down-sloping orbitals have positive prolate single particle quadrupole moments and up-sloping orbitals have negative oblate quadrupole moments. Jerry Garret et al. [32] have shown that the high- $K$ upsloping $v[505] 11 / 2^{-}$"flying fish" orbital does not partake in the normal monopole pairing (see text). The density of down-sloping orbitals is much greater than that of the extruded up-sloping orbitals.

Clearly the actinides and nuclei near $\mathrm{N}=90$ are not the only regions of the nuclear chart where pairing isomers can occur. They will be located at the start of regions of deformation, where the increasing number of protons or neutrons in low $\Omega$ aligned orbits, which increase the nuclear deformation, force high $\Omega$ orbitals, from the filled lower shell, to the Fermi surface. Examples would be near ${ }^{128}{ }_{58} \mathrm{Ce}_{70}$ where the neutrons are at mid-shell, causing a strong deformation, and there are 8 protons outside the closed $\mathrm{Z}=50$ shell. In this case the "flying fish" orbital forced to the Fermi Surface will be the proton $\pi[404] 9 / 2^{+}$orbital. Another region is near ${ }^{102}{ }_{44} \mathrm{Ru}_{58}$ where the deformation brings both the $\pi[404] 9 / 2^{+}$proton orbital and the $v[404] 9 / 2^{+}$neutron orbitals close to the Fermi surface. 


\section{5. $\quad \mathrm{K}^{\pi}=2^{+}$Collective bands}

In all well deformed prolate even-even nuclei, from ${ }^{22} \mathrm{Ne}$ to the actinides, $\mathrm{K}^{\pi}=2^{+}$bands are observed at low excitation energies well within the pairing gap. Usually the $\mathrm{K}^{\pi}=2^{+}$bandheads are at lower energies than the $\mathrm{O}_{2}{ }^{+}$states. Single particle transfer gives information on the quasi-particle/hole structure of any states not in the ground state band of the final nucleus. States that are strongly populated will consist of the target odd quasi-particle coupled to some other quasiparticle. Assuming axially symmetric states, $\mathrm{K}$ will be a good quantum number, and any $\mathrm{p}$-h component of the $\gamma$-band configuration should be composed of quasi-particles in Nilsson orbits $\left[\mathrm{Nn}_{\mathrm{z}} \Lambda\right] \Omega$ of the same parity and where $\Delta \mathrm{K}=\left|\Omega_{\text {target }} \pm \Omega_{\text {transfer }}\right|=2$. The (d,p) reaction has been used to populate states in ${ }^{158} \mathrm{Gd},{ }^{164} \mathrm{Dy},{ }^{172} \mathrm{Yb}$ and ${ }^{173} \mathrm{Yb}$ [35]. A straightforward calculation using Nilsson wavefunctions gives a good account of the relative strengths of the populations of the ground state and $\gamma$ bands in all four nuclei. In these cases the configurations involved have $\Delta \mathrm{K}=2$. Similarly the ${ }^{151} \mathrm{Sm}(\mathrm{d}, \mathrm{p}){ }^{152} \mathrm{Sm}$ reaction [36] strongly populates the $\gamma$ band as the ground state of ${ }^{151} \mathrm{Sm}$ is [523]5/2 and the [521]1/2 orbital, giving $\Delta \mathrm{K}=2$, is available above the Fermi Surface. In contrast, the neutron pick-up reaction ${ }^{151} \mathrm{Sm}(\mathrm{p}, \mathrm{d}){ }^{150} \mathrm{Sm}$ does not populate the $\gamma$ band [36] as there is no suitable $\Delta K=2$ orbital to couple to below the Fermi Surface. Proton stripping reactions to ${ }^{154} \mathrm{Gd}$ using the $\left({ }^{3} \mathrm{He}, \mathrm{d}\right)$ and $(\alpha, \mathrm{t})$ reactions [37] populate the $\gamma$ band. The target nucleus ${ }^{153} \mathrm{Eu}$ has its odd proton in the $[431] 5 / 2^{+}$orbit and the $\Delta K=2$ orbit [411]1/2+ is just above the Fermi Surface. Again, in contrast, the $(\mathrm{t}, \alpha)$ proton pick-up reaction to the nuclei ${ }^{152} \mathrm{Sm}[38],{ }^{164} \mathrm{Dy}[39]$ and ${ }^{174} \mathrm{Yb}$ [40] do not populate the $\gamma$ bands at all. Again this is because there are no suitable $\Delta \mathrm{K}=2$ orbitals below the Fermi Surface. The (p,t) pick-up reactions usually populate the $\gamma$ band very weakly.

In the rotation-vibration model [41] the energies of $\beta$ and $\gamma$ vibrations are given, in an obvious notation, by

$$
\operatorname{Ex}\left(\mathbf{n}_{\beta} n_{\gamma} I K\right)=\hbar \omega_{\beta}\left(n_{\beta}+1 / 2\right)+\hbar \omega_{\gamma}\left(2 n_{\gamma}+1 / 2|K|+1\right)+\left[I(I+1)-K^{2}\right] \hbar^{2} / 2 \mathscr{g}
$$

Most text books manage to miss the $1 / 2|\mathbf{K}|$ term in Equ. 3, giving the impression that the first $\mathrm{K}^{\pi}$ $=2^{+}$band has $\mathrm{n}_{\gamma}=1$. But Equ. 3 shows that the traditional $\mathrm{K}^{\pi}=2^{+} \gamma$-band is not a band containing a quantum $\hbar \omega_{\gamma}$ in the $\gamma$ direction but has $\mathrm{n}_{\gamma}=0$ and a bandhead excitation energy given by $\mathrm{E}_{\mathrm{x}}=$ $\left(\hbar \omega_{\gamma}+\hbar^{2} / \mathscr{g}\right)$. These bands are referred to by Davydov as "anomalous rotational bands" [42]. In the rotation-vibration model there is a strong coupling between rotations and $\gamma$-vibrations, physically expressing the fact that rotations with non-vanishing $\mathrm{K}$ become possible only in the presence of dynamical triaxial deformation [41]. Any model having the $\gamma$ degree of freedom will have zeropoint fluctuations and a similar origin for $\mathrm{K}^{\pi}=2^{+}$bands. In Fig. 6 we show the systematics for the experimental values of the $\hbar \omega_{\gamma}$ "phonon" quantum, calculated using Equ. 3, for $\mathrm{K}=2^{+}$bands between $\mathrm{Z}=60-70$ and $\mathrm{N}=88-98$. The values of $\hbar \omega_{\gamma}$ vary smoothly with $\mathrm{Z}$ and $\mathrm{N}$ unlike the systematics for the excitations energies of the $0_{2}{ }^{+}$states, equal to $\hbar \omega_{\beta}$ if Equ. 3 is used, shown in Fig. 4. The levels of $\mathrm{K}^{\pi}=2^{+}$bands have both even and odd spins and are often divided into even $2+$, $4+, 6+\ldots$ (natural parity) and odd $3+, 5+, 7+\ldots$ (unnatural parity) spin levels for clarity. The even spin levels decay to the levels in the ground state band (gsb) by not only $\Delta \mathrm{J}=2$ transitions, but also $\Delta \mathrm{J}=0$ and $\Delta \mathrm{J}=-2$ transitions. As $\Delta \mathrm{K}=2$ in these out-of-band transitions, $\mathrm{M} 1$ components are K-forbidden in the $\Delta J=0 \gamma$-rays. Similarly the $\Delta J= \pm 1$ transitions from the odd spin members to the ground state band will be mostly E2 and contain very small M1 components at most $[43,44]$. Generally in-band $\Delta \mathrm{J}=1 \mathrm{M} 1$ transitions between the even and odd spin members of $\gamma$-bands are very weak [42]. This means that $\mathrm{g}_{\mathrm{K}} \approx \mathrm{g}_{\mathrm{R}}$ for $\gamma$-bands. The E2 transition strengths are always of the order of 10W.u. (Weisskopf single particle units). 


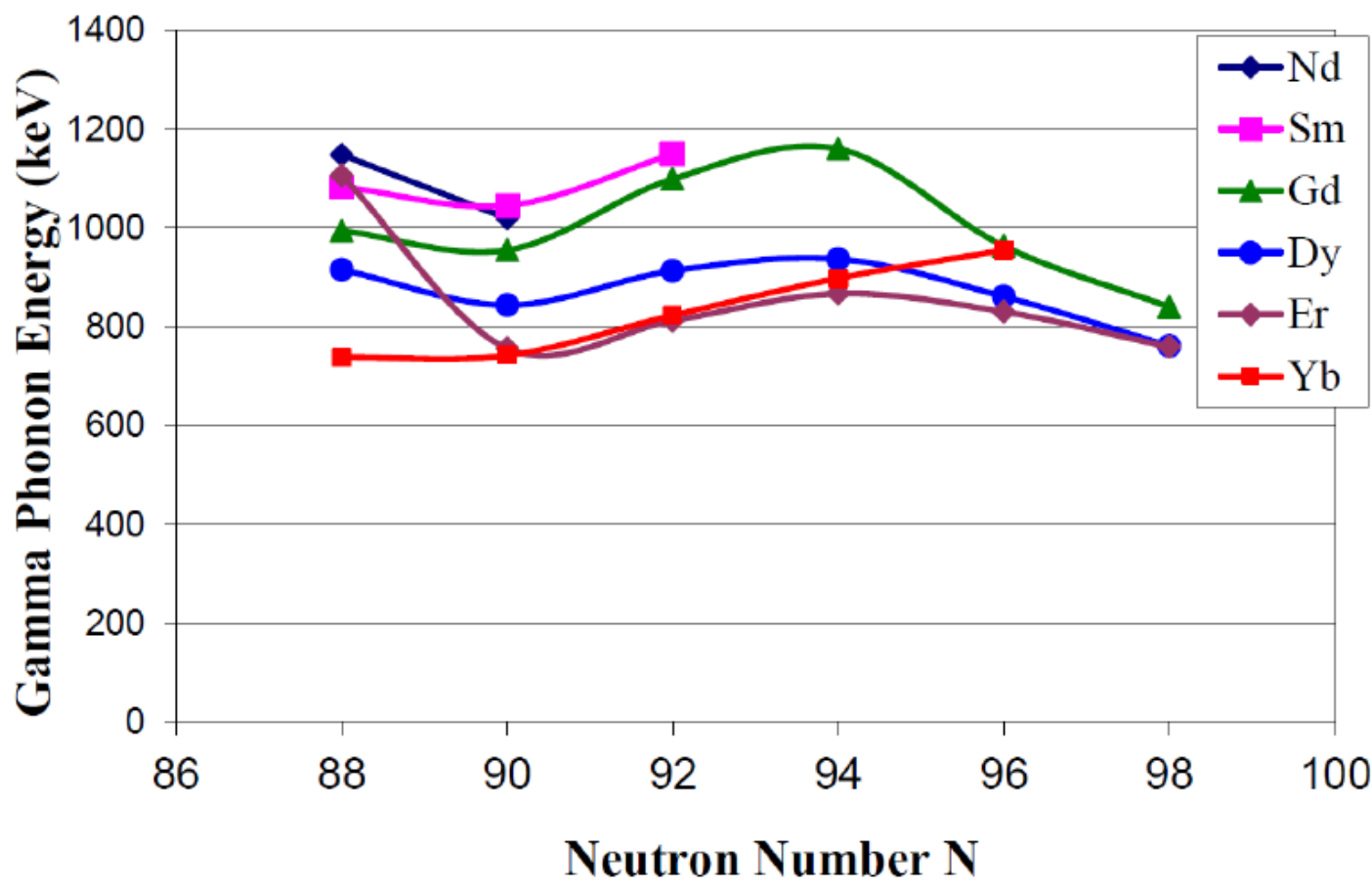

Fig. 6. Systematics of $\gamma$ phonon energy $\hbar \omega_{\gamma}$ for $N=86-98$ and $Z=60-70$ calculated using Equ. 3 .

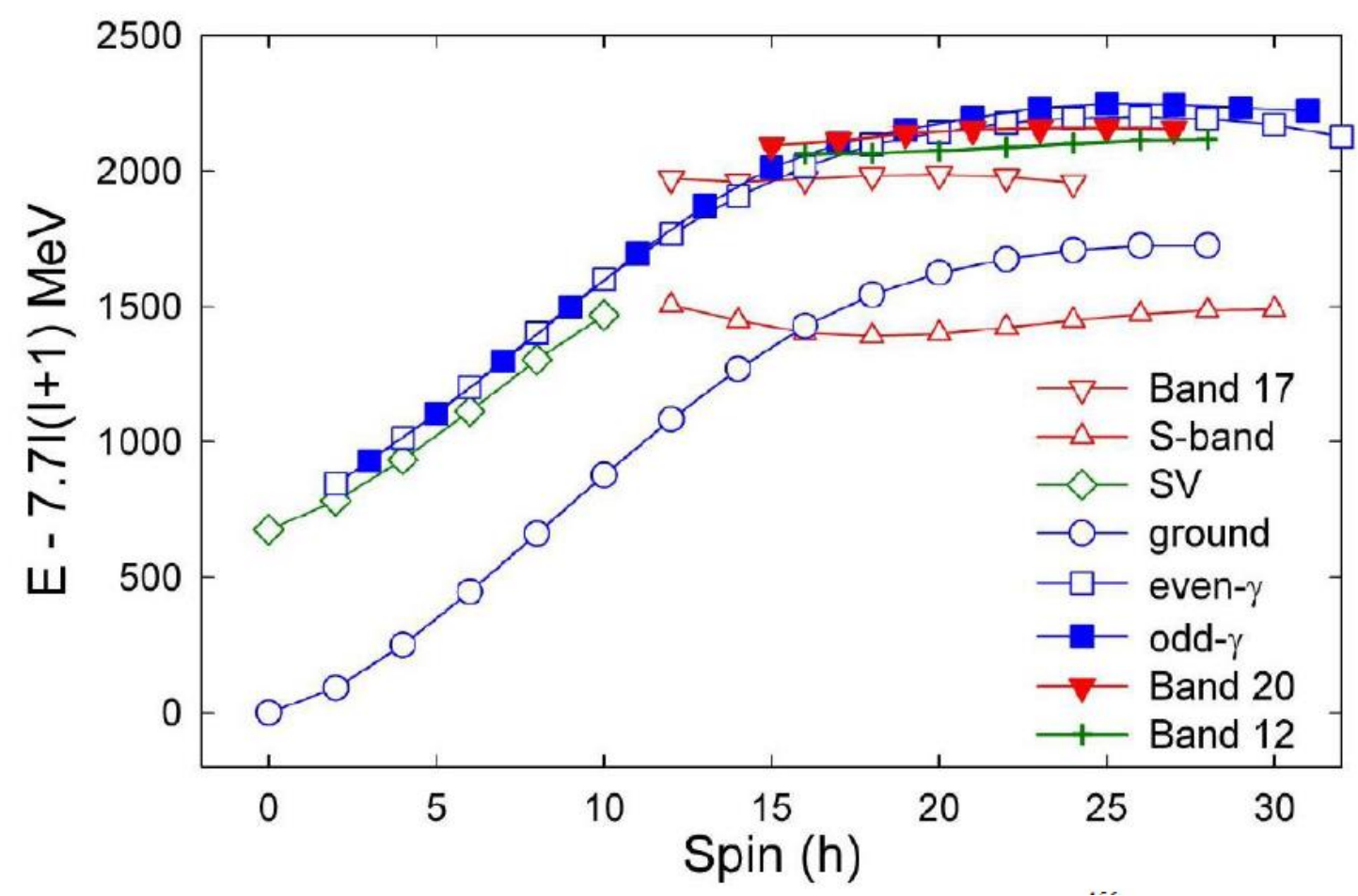

Fig. 7. Plot of the excitation energy, minus a rigid rotor, for the positive-parity bands in ${ }^{156} \mathrm{Dy}$ [44]. The Second Vacuum (SV) band is the $0_{2}{ }^{+}$band which is interpreted here to be a "pairing isomer" (see text).

It is not very usual for $\gamma$-bands to be identified much above spin $12^{+}$as they are usually about 1.0 MeV above the yrast line. This makes it difficult to populate such states in fusion-evaporation $(\mathrm{HI}, \mathrm{xn})$ reactions as they are embedded in other structures which compete for intensity. The use of very heavy ion beams to Coulomb excite the most deformed nuclei has, in favourable cases, 
allowed $\gamma$ bands to be traced up to much higher spins. A notable feature of $\gamma$ bands is that they track the intrinsic configuration, usually the ground state, they are based on. An example of this is shown in Fig. 7 for the $\gamma$ band in ${ }^{156}$ Dy [45]. Here both signatures of the $\gamma$ band track the ground state configuration up to its highest observed spin of $32^{+}$. The aligned band in ${ }^{156} \mathrm{Dy}$, which has a band crossing with the band based on the $0_{2}{ }^{+}$state [46], shows no sign of any interaction with the $\gamma$ band. The $\gamma$ band has a small signature splitting at higher spins.

A spectacular example of a $\gamma$ band built on sequential alignments has been found in ${ }^{160} \mathrm{Er}$ by Ollier et al. [47]. Only the odd spin members of the $\gamma$ band are seen, but they extend up to spin $43^{+}$and track the yrast states around both the $v\left(i_{13 / 2}\right)^{2} \mathrm{AB}$ neutron alignment and then around the $\pi\left(h_{11 / 2}\right)^{2}$ proton alignment (second back-bend). Again the data indicate that the crossings of the $\gamma$ band come at slightly lower spins and frequencies than in the yrast states. The data is quite remarkable. It shows that on every intrinsic or aligned configuration a $\gamma$-band is built with an added $(\mathrm{K}+2)$ quantum number. Whatever the $\gamma$ bands are built with, it does not seem to be affected by the configurations causing the alignments. Both even and odd spin members of positive parity bands, that we have interpreted as a $\gamma$ band built on top of the aligned $S$ band in ${ }^{156} \mathrm{Dy}$, are shown in Fig. 7 as bands 17 and 20 [45].

Just as in the case of $\mathrm{O}_{2}{ }^{+}$states, a strong test of any theory of $\gamma$ vibrations is the experimental evidence of how single particle states couple to the core $\gamma$ vibration. Each single particle state with Nilsson quantum number $\Omega$ can couple to the core $\mathrm{K}^{\pi}=2^{+}$excitation either in a parallel mode to give $K_{>}=(\Omega+2)$ or in an anti-parallel mode to give $K_{<}=|\Omega-2|$. There can be a splitting of the bandheads of these two bands which will give information on the particle-core excitation interaction. Clearly the band with $\mathrm{K}$, will usually be nearer yrast and therefore easier to detect in (HI,xn) reactions. However the $\mathrm{K}<$ band can be found, when $\mathrm{K}<$ is small, in experiments such as $(\mathrm{n}, \gamma)$ and $\left(n, n^{\prime} \gamma\right)$ experiments [17]. The most complete data sets on the coupling of the ground state nucleon in an odd nucleus to a core $\mathrm{K}^{\pi}=2^{+}$excitation are Coulomb excitation experiments [48] on ${ }^{165}{ }_{67} \mathrm{Ho}_{98}$ and ${ }^{167}{ }_{68} \mathrm{Er}_{99}$, which share the core nucleus ${ }_{68}^{166} \mathrm{Er}_{98}$, and fission fragment spectroscopy $[49,50]$ on the trio ${ }^{103}{ }_{41} \mathrm{Nb}_{62},{ }^{104}{ }_{42} \mathrm{Mo}_{62}$ and ${ }^{105}{ }_{42} \mathrm{Mo}_{63}$.

\section{6. $\quad y$ Vibrations or $y$ Deformation?}

As the data indicate that any time-dependent " $\beta$ vibrations" are at higher excitation energies than formerly imagined, then we might well expect any " $\gamma$ vibrations" to also be well above the pairing gap? In that case we are left with the other explanation given by Bohr and Mottelson that the plethora of $\mathrm{K}^{\pi}=2^{+}$bands found in deformed nuclei throughout the nuclear chart are due to these nuclei not being axially symmetric [1] (page $166 \mathrm{Vol}$. II). The way of deciding between $\gamma$ vibrations and axial asymmetry is to look experimentally for the predicted doublet of two-phonon $\mathrm{K}^{\pi}=0_{\gamma \gamma}{ }^{+}$and $4_{\gamma \gamma}{ }^{+}$bands for vibrations or the lone $\mathrm{K}^{\pi}=4_{\gamma \gamma}{ }^{+}$band predicted for axial asymmetry. This is difficult, as these structures are even further from the yrast line and will usually be embedded in a high density of $\mathrm{p}-\mathrm{h}$ states with which they will mix. The experimental data to date are not very convincing, [51] and references therein.

Clearly it will be experimentally easiest to detect the $\mathrm{K}^{\pi}=4_{\gamma \gamma}{ }^{+}$bands as these will be nearest yrast. Also it will be best to look where the $\mathrm{K}^{\pi}=2^{+}$bands have the lowest excitation energy. The lowest $\mathrm{K}^{\pi}=2^{+}$bands are in the Os nuclei [52-54]. These nuclei also have low-lying $\mathrm{K}^{\pi}=4^{+}$bands at about twice the excitation energy of the $\mathrm{K}^{\pi}=2^{+}$bands, making them good candidates for being a member of a two-phonon doublet. There is a particularly complete set of Coulomb excitation (Coulex) data on the ${ }^{186,188,190,192}$ Os isotopes measured by Wu et al. [53] giving both diagonal and 
off-diagonal matrix elements for the $\mathrm{K}^{\pi}=2^{+}$bands and the $\mathrm{K}^{\pi}=4^{+}$bands. These authors conclude that the $\mathrm{K}^{\pi}=4^{+}$states are consistent with them being two- phonon $\gamma$-vibration excitations $4{ }_{\gamma \gamma}{ }^{+}$. This conclusion gave rise to a degree of robust discussion! It was pointed out by Dennis Burke [55] that the conclusion of [53] rests on consideration of the matrix elements measured in their Coulex experiment and did not consider transfer and inelastic scattering experiments. The proton pick-up reaction ${ }^{191} \operatorname{Ir}(\mathrm{t}, \alpha){ }^{190} \mathrm{Os}$ [56] and the proton stripping reactions ${ }^{185,187} \operatorname{Re}\left({ }^{3} \mathrm{He}, \mathrm{d}\right){ }^{186,188} \mathrm{Os}$ [57] show that the candidate two-phonon $4{ }_{\gamma \gamma}{ }^{+}$states in Os have a very strong component of the $1 \mathrm{p}-1 \mathrm{~h}$ proton configuration $\left\{\pi[402] 3 / 2^{+}+\pi[402] 5 / 2^{+}\right\}$. They are also strongly populated in (p,p') and $\left(\alpha, \alpha^{\prime}\right)$ with $L=4$ angular distributions [58-60]. The final conclusion [61,62] was that the $\mathrm{K}^{\pi}=4^{+}$ state in ${ }^{190} \mathrm{Os}$ at $1163 \mathrm{keV}$, almost exactly twice the excitation energy of the $\mathrm{K}^{\pi}=2^{+}$state at 557 $\mathrm{keV}$, is a highly mixed state with strong proton $1 \mathrm{p}-1 \mathrm{~h}$ and hexadecapole components. In addition, the $\Delta \mathrm{I}=1$ in-band transitions from the $5^{+}$and $6^{+}$states for the $\mathrm{K}^{\pi}=4^{+}$band are strong. For most $\mathrm{K}^{\pi}=2^{+}$bands the $\Delta \mathrm{I}=1$ in-band transitions are very weak or undetected, which is expected for both $\gamma$ vibrational and $\gamma$ deformed scenarios. Again the data mitigates against the $1163 \mathrm{keV}$ state being the bandhead of a two quadrupole phonon collective excitation. If this $\mathrm{K}^{\pi}=4^{+}$state at $1163 \mathrm{keV}$ is very mixed, there has to be at least one higher $\mathrm{K}^{\pi}=4^{+}$state to mix with. The next possible $4^{+}$ state in the data tables is at $1708 \mathrm{keV}$ with spin assignments of $\left(2^{+}, 3^{+}, 4^{+}\right)$. This means that the unmixed excitation of a $\mathrm{K}^{\pi}=4_{\gamma \gamma}{ }^{+}$two-phonon band has to be well above twice the one-phonon excitation energy. This definitely ruins the elegant " $\gamma$ vibration" interpretation of the structure of ${ }^{190}$ Os. Allmond et al. [54] have revisited the extensive Coulex data of Ref. [53] and reanalysed it in terms of their triaxial rotor model with independent inertia and electric quadrupole tensors [63]. They conclude that their model gives an improved description of the large set of E2 matrix elements except for those measured for the candidate two-phonon $\mathrm{K}^{\pi}=4_{\gamma \gamma}{ }^{+}$band. They point out that "the success of this model with respect to the $K^{\pi}=0^{+}$band and $K^{\pi}=2^{+}$band is insufficient to necessitate the triaxial features of the model" and that "there must be (at least) a second $K^{\pi}=4^{+}$band which carries the missing \{E2\} strength". The partner two-phonon $\mathrm{K}^{\pi}=0_{\gamma \gamma}{ }^{+}$band to the $\mathrm{K}^{\pi}=4_{\gamma \gamma}{ }^{+}$ band, that has to exist in any vibrational description of ${ }^{190} \mathrm{Os}$, gets little attention in all the experiments and analysis.

Similar $\mathrm{K}^{\pi}=4^{+}$bands exist in the nuclei near $\mathrm{N}=90$ [51,64-67] that have been proposed to be two $\gamma$ phonon states. In these nuclei the $\mathrm{K}^{\pi}=2^{+}$bandheads are at excitation energies above 750 $\mathrm{keV}$. The proposed two phonon $\mathrm{K}^{\pi}=4_{\gamma \gamma}{ }^{+}$states are not at such elegantly twice the one-phonon energies as for the Os isotopes. This is usually ascribed to the $\sim 1600 \mathrm{keV}$ energies being nearer the top of the pairing gap leading to increased $\mathrm{p}-\mathrm{h}$ mixing. An example is the $\mathrm{K}^{\pi}=4^{+}$bandhead at $1646 \mathrm{keV}$ in ${ }^{154}{ }_{64} \mathrm{Gd}_{90}[66,67]$ which is at an excitation energy 1.65 times the excitation energy of the $\mathrm{K}^{\pi}=2^{+}$bandhead. The state is strongly populated in (d,d') experiments $[68,69]$ with $\mathrm{L}=4$ and in proton stripping [51] leading to a preferred proton $\mathrm{p}$ - $\mathrm{h}$ configuration $\left\{\pi[413] 5 / 2^{+}+\pi[411] 3 / 2^{+}\right\}$. Also the band built on the $\mathrm{K}^{\pi}=4^{+}$state has been established up to spin $13^{+}$and is connected by $\Delta \mathrm{I}=1$ strong in-band $\mathrm{M} 1$ transitions [70]. The $\mathrm{K}^{\pi}=2^{+}$bands always have extremely weak, or undetectable, $\Delta \mathrm{I}=1$ in-band transitions which is expected on any collective model. There is no reason either to expect strong $\Delta \mathrm{I}=1$ in-band transitions in $4_{\gamma \gamma}{ }^{+}$bands. In short, a two-phonon structure has been misidentified for these states.

There is one reasonable claim for identifying the $0_{\gamma \gamma}{ }^{+}$member of a two phonon doublet. In ${ }^{166} \mathrm{Er}$ a $\mathrm{K}^{\pi}=4^{+}$state at $2055 \mathrm{keV}$ decays strongly to the $\mathrm{K}^{\pi}=2^{+}$band. A level at $1943 \mathrm{keV}$ has been shown to be a $0^{+}$state which has enhanced E2 decays to the $\mathrm{K}^{\pi}=2^{+}$band and is therefore a best candidate for a $0_{\gamma \gamma}{ }^{+}$level [71]. 


\section{Conclusions}

The invention of $\mathrm{Ge}(\mathrm{Li})$ detectors [72-74] by Ewan and Tavendale, which have developed into the present day large arrays of escape-suppressed HPGe photon detectors, has seduced many of us into over emphasizing the many nuclear properties which can be obtained using $\gamma$-ray spectroscopy. The establishment of complex decay schemes and the measurement of $B(E \lambda)$ for many levels are insufficient to necessarily pin down the essential physics determining the structure of excited nuclei. There is still a need for particle transfer data to identify single particle structures and for inelastic scattering data to point at collective phenomena. The data firmly establish that the $0_{2}{ }^{+}$states, in the pairing gap of even-even nuclei that are usually labelled as " $\beta$ vibrations", are nothing of the kind. They are seniority zero states lowered into the pairing gap by configuration dependent pairing. This has cast doubt on the identification of the first excited $\mathrm{K}^{\pi}=2^{+}$bands in all deformed nuclei as arising from time-dependent " $\gamma$ vibrations" of the nuclear mean field. These $\mathrm{K}^{\pi}=2^{+}$bands can equally be due to the $\gamma$ degree of freedom allowing asymmetry of the quadrupole shape. The inconclusive data on proposed two phonon structures points us to look for other descriptions of collective excitations. If deformed and spherical nuclei do not have timedependent vibrations of the nuclear shape to give rise to the observed low energy quadrupole excitations, then what about the lowest negative parity states usually identified as "octupole vibrations"? If the $\mathrm{K}^{\pi}=2^{+}$bands are due to the breaking of axial symmetry, could the lowest negative parity bands be due to the breaking of reflection symmetry? To push the analogy further; are the low excitation energy $\mathrm{K}^{\pi}=4^{+}$states due to a hexadecapole component of the nuclear mean field? We are going to be stuck with the nuclear shape looking like

$$
\mathrm{R}=\mathrm{R}_{0}\left(1+\Sigma \mathrm{a}_{2, \mu} \mathrm{Y} 2, \mu+\Sigma \mathrm{a}_{3}, \mu \mathrm{Y}_{3, \mu}+\Sigma \mathrm{a} 4, \mu \mathrm{Y}_{4, \mu}+\ldots .\right)
$$

Although this approach simplifies the physics by doing away with time-dependent vibrations, phonons, bosons and the like, it leaves us with a formidable mixture of shapes.

\section{Acknowledgements}

I am delighted to thank Sven Áberg, Mitch Allmond, Peter Butler, Paul Garrett, David Kulp, Eddie Paul, Mark Riley, David Rowe, John Simpson and John Wood for very enlightening discussions. I greatly appreciate the assistance, encouragement and comradeship of many colleagues from iThemba Laboratory for Accelerator Based Sciences (LABS) and from the Universities of the Western Cape and of Johannesberg, in particular Rob Bark, Suzan Bvumbi, Tshepo Dinoko and Siyabonga Majola. I am thankful for some funding from the South African National Research Foundation.

\section{References}

[1] Aage Bohr and B. R. Mottelson, Nuclear structure, Vols. I \& II, (Benjamin 1975)

[2] George Gamow, in Proc. Roy. Soc. Lon. A136, 386 (1929)

[3] N. Bohr, Nature 137, 344, 351 (1936)

[4] N. Bohr and F. Kalckar, Dan. Mat. Fys. Medd. 14, no.10 (1937)

[5] Lord Rayleigh, Proc. Roy. Soc. of London 29, 71 (1879) App. II, Equ. (40)

[6] Leon van Dommelen, "Quantum Mechanics for Engineers" 
www.eng.fsu.edu/ dommelen/quantum/style_a/nt_liqdrop.html

[7] C. F. von Weizsäcker, Zeit. für Phys. 96, 431 (1935)

[8] V. M. Strutinski, Nucl. Phys. A95, 420 (1967)

[9] V. M. Strutinski, Nucl. Phys. A122, 1 (1968)

[10] S. Flügge, Ann. Phys. Lpz. 431, 373 (1941)

[11] Aage Bohr, Dan. Mat. Fys. Medd. 26, no.14 (1952)

[12] P. M. A. Dirac, Proc. Roy. Soc. A133, 621 (1927)

[13] B. Podolsky, Phys. Rev. 32, 812 (1928)

[14] P. E. Garrett, J. Phys. G27, R1 (2001)

[15] W. D. Kulp et al., Phys. Rev. C77, 061301(R) (2008)

[16] M. A. M. Shahabuddin et al; Nucl. Phys. A340, 109 (1980)

[17] H. H. Schmidt et al., J. Phys. G12, 411 (1986)

[18] G. Løvhøiden, J. C. Waddington, K. A. Hagemann, S. A. Hjorth and H. Ryde, Can J. Phys. 51, $1369(1973)$

[19] J. F. Sharpey-Schafer et al., Eur. J. Phys. A47, 6 (2011)

[20] W. D. Kulp et al., Phys. Rev. C76, 034319 (2007) and private communication.

[21] J. F. Sharpey-Schafer et al., Eur. J. Phys. A47, 5 (2011)

[22] T. S. Dinoko, PhD Thesis, Univ. of Western Cape (2014)

[23] R. A. Bark et al., private communication

[24] W.-T. Chou, Gh Cata-Danil, N. V. Zamfir, R. F. Casten and N. Pietralla, Phys. Rev. C64, $057301(2001)$

[25] J. V. Maher, J. R. Erskine, A. M. Friedman, J. P. Schiffer and R. H. Siemssen, Phys. Rev. Lett. $25,302(1970)$

[26] R. F. Casten et al., Phys. Lett. 40B, 333 (1972)

[27] R. E. Griffin, A. D. Jackson and A. B. Volkov, Phys. Lett. 36B, 281 (1971)

[28] Shiro Yoshida, Nucl. Phys. 33, 685 (1962)

[29] S. K. Abdulvagabova, S. P. Ivanova and N. I. Pyatov, Phys. Lett. 38B, 251 (1972)

[30] W. I. van Rij and S. H. Kahana, Phys. Rev. Lett. 28, 50 (1972)

[31] D. R. Bès, R. A. Broglia and B. Nilsson, Phys. Lett. 40B, 338 (1972) 
[32] I. Ragnarsson and R. A. Broglia, Nucl. Phys. A263, 315 (1976)

[33] J. D. Garrett et al., Phys. Lett. B118, 297 (1982)

[34] J. M. Rees et al., Phys. Rev. C83, 044314 (2011)

[35] W. N. Shelton and C. E. Watson, Phys. Lett. 22, 648 (1966)

[36] D. E. Nelson, D. G. Burke, J. C. Waddington and W. B. Cook, Can. J. Phys. 51, 200 (1975)

[37] D. G. Burke, J. C. Waddington and O. P. Jolly, Nucl. Phys. A688, 716 (2001)

[38] C. R. Hirning and D. G. Burke, Can. J. Phys. 55, 2288 (1977)

[39] S. J. Freeman et al., Nucl. Phys. A554, 333 (1993)

[40] S. J. Freeman et al., Nucl. Phys. A552, 10 (1993)

[41] W. Greiner and J. A. Maruhn, Nuclear Models, (Springer 1996) p158/9

[42] A. S. Davydov, Atomic Energy Review 6 No2, 3 (1968)

[43] K. Schreckenbach and W. Gelletly, Phys.Lett. 94B, 298 (1980)

[44] I. Alfter, E. Bodenstedt, W. Knichel and J. Schüth, Nucl. Phys. A635, 273 (1998)

[45] S. N. T. Majola et al., Phys. Rev. C91, 034333 (2015)

[46] D. Ward, R. L. Graham, J. S. Geiger and H. R. Andrews; Phys. Lett. 44B, 39 (1973)

[47] J. Ollier et al., Phys. Rev. C83, 044309 (2011)

[48] G. Gervais et al., Nucl. Phys. A624, 257 (1997)

[49] Jian-Guo Wang et al., Nucl. Phys. A834, 94c (2010)

[50] J. B. Snyder et al., Phys. Lett. B723, 61 (2013)

[51] D. G. Burke, Phys. Rev. Lett. 73, 1899 (1994)

[52] T. Kibédi, G. D. Dracoulis, A. P. Byrne, P. M. Davidson and S. Kuyucak, Nucl. Phys. A567, $183(1994)$

[53] C. Y. Wu et al., Nucl. Phys. A607, 178 (1996)

[54] J. M. Allmond, R. Zaballa, A. M. Oros-Peusquens, W. D. Kulp and J. L. Wood, Phys. Rev. C78, 014302 (2008)

[55] D. G. Burke, Phys. Lett. B406, 200 (1997)

[56] R. D. Bagnal et al., Phys. Rev. C20, 42 (1979)

[57] A. A. Phillips et al., Phys. Rev. C82, 034321 (2010)

[58] F. Todd Baker et al., Nucl. Phys. A501, 546 (1989) 
[59] D. G. Burke et al., Phys. Lett. B78, 48 (1978)

[60] F. Todd Baker, Nucl. Phys. A371, 68 (1981)

[61] D. G. Burke, Phys. Rev. C66, 039801(2002)

[62] C. Y. Wu et al., Phys. Rev. C66, 039802 (2002)

[63] J. L. Wood, A. M. Oros-Peusquens, R. Zaballa and J. M. Allmond, Phys. Rev. C70, 024308 (2004)

[64] H. G. Börner et al., Phys. Rev. Lett. 66, 691 (1991)

[65] M. Oshima et al., Nucl. Phys. A557, 635c (1993)

[66] X. Wu, A. Aprahamian, J. Castro-Ceron and Cyrus Baktash, Phys. Lett. B316, 235 (1993)

[67] X. Wu, A. Aprahamian, S. W. Fisher, W. Reviol, G. Liu and J. X. Saladin, Phys. Rev. C49, $1837(1994)$

[68] D. C. Sousa, L. L. Riedinger, E. C. Funk and J. W. Mihelich, Nucl. Phys. A238, 365 (1975)

[69] R. L. West, E. C. Funk and J. W. Mihelich, Phys. Rev. C18, 679 (1978)

[70] J. F. Sharpey-Schafer et al., Proc. Int. Conf. on Frontiers in Nuclear Structure, Astrophysics and Reactions (FINUSTAR2) Crete, Greece. Sept 2007, AIP Con. Proc. 1012 p19

[71] P. E. Garrett, M. Kadi, Min Li, C. A. McGrath, V. Sorokin, Minfang Yeh and S.W. Yates, Phys. Rev. Lett. 78, 4545 (1997)

[72] A. J. Tavendale and G. T. Ewan, Nucl. Instr. Meth. 25, 185 (1963)

[73] G. T. Ewan and A. J. Tavendale, Nucl. Instr. Meth. 26, 183 (1964)

[74] G. T. Ewan and A. J. Tavendale, Can. J. Phys. 42, 2286 (1964) 